\title{
Anaplastic lymphoma receptor tyrosine kinase-negative inflammatory myofibroblastic tumor of triceps brachii: Case report
}

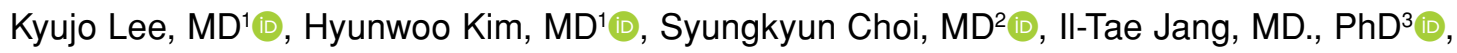 \\ Hyunjin Lee, MD ${ }^{1}$ (1)
}

${ }^{1}$ Nanoori 2bko R\&D Institute, Nanoori Hospital Suwon, Suwon, Republic of Korea

${ }^{2}$ Department of Orthopedic Surgery, Nanoori Hospital Gangnam, Gangnam, Republic of Korea

${ }^{3}$ Department of Neurosurgery, Nanoori Hospital Gangnam, Gangnam, Republic of Korea

Inflammatory myofibroblastic tumor (IMT) is a rare benign tumor with an unknown cause and comprised of plasma cells, lymphocytes, histocytes, and vascular tissue. First discovered in 1954 by Umiker and Iverson, ${ }^{[1]}$ Inflammatory myofibroblastic tumors sometimes show similar manifestations as malignant tumors. They usually occur in the lungs and upper airways, and cases in areas such as the head and neck, liver, spleen, pancreas, gastrointestinal tract, posterior peritoneum, heart, kidneys, bladder, uterus, lymph nodes, and skin have also been reported..$^{[2,3]}$ There are some reports of IMT on thigh in musculoskeletal system. ${ }^{[4,5]}$ Histopathologically, they are composed of inflammatory and spindle cells; hence, they are named IMTs. The course of these tumors cannot be

Received: March 30, 2020

Accepted: May 18, 2020

Published online: July 16, 2020

Correspondence: Hyunjin Lee, MD. Nanoori 2bko R\&D Institute, Nanoori Hospital Suwon, 295, Jungbu-daero, Yeongtong-gu, 16503 Gyeonggi-Do, Republic of Korea.

E-mail: 0083ackmong@naver.com

Doi: $10.5606 /$ ehc. 2020.74662

Citation: Lee K, Kim H, Choi S, Jang II-T, Lee H. Anaplastic lymphoma receptor tyrosine kinase-negative inflammatory myofibroblastic tumor of triceps brachii: Case report. Jt Dis Relat Surg 2020;31(3):605-609.

O2020 All right reserved by the Turkish Joint Diseases Foundation

This is an open access article under the terms of the Creative Commons Attribution-NonCommercial License, which permits use, distribution and reproduction in any medium, provided the original work is properly cited and is not used for commercial purposes (http://creativecommons.org/licenses/by-nc/4.0/).

\section{ABSTRACT}

Inflammatory myofibroblastic tumor (IMT) is a non-neoplastic benign lesion comprising various inflammatory cells, including myofibroblasts and vascular tissues. It is a rare tumor that sometimes shows similar signs and progression as malignant tumors. The anatomical sites of IMTs include the lungs, liver, orbit, skin, mesentery, and maxillary sinus, but they rarely occur in the limb musculoskeletal system. To our knowledge, no case of neurological symptoms caused by the tumor in the triceps brachii muscle has been reported. In this article, we report the case of a 42-year-old male patient with an IMT that grew rapidly in the triceps brachii muscle and consequently caused symptoms of ulnar nerve lesion owing to its increasing size. The patient showed no ulnar nerve lesion symptoms after undergoing wide excision and was diagnosed with anaplastic lymphoma receptor tyrosine kinasenegative IMT.

Keywords: Anaplastic lymphoma receptor tyrosine kinase-negative inflammatory myofibroblastic tumor, triceps brachii.

accurately predicted. Depending on the research, they are called "inflammatory pseudotumors", "benign myofibroblastoma" and "inflammatory fibrosarcoma" among other names, making the choice of treatment difficult. Although the exact cause is unknown, the rearrangement of the chromosomal band 2p23, in which the anaplastic lymphoma kinase (ALK) gene is located, is linked to approximately 50\% of the tumors. On the basis of recent reports that have classified them as clinically malignant tumors, we recommend that they be treated as true tumors and not pseudotumors. ${ }^{[6]}$ Full recovery has been reported to be attainable via complete resection. Other reports described disease improvement after administration of steroidal or non-steroidal anti-inflammatory 
medication. ${ }^{[7]}$ Moreover, symptoms subsided after chemotherapy with drugs such as crizotinib and clarithromycin. ${ }^{[8,9]}$ In this article, we report the case of an ALK-negative IMT that showed symptoms of an ulnaris nerve lesion, with the tumor occurring and growing rapidly in the triceps brachii muscle, within the limb musculoskeletal system, before improvement of the symptoms with surgical treatment. ${ }^{[10]}$

\section{CASE REPORT}

The patient was a 42-year-old male who was admitted for a rapidly growing mass in the left posterior humeral area first palpated one month before admission. The patient reported dropping objects when lifting with the left hand and desensitization on the left side, with motor strength grade IV. He had no specific findings in the past or relevant family history. His laboratory results were normal. On physical examination, the left side showed signs of desensitization, numbness in the ulnar nerve area, hand vibrations during fine motions, and decreased grip strength to motor strength grade IV+. The tumor was firm and non-movable during palpation and showed a positive Tinel sign. On the upper arm magnetic resonance imaging performed

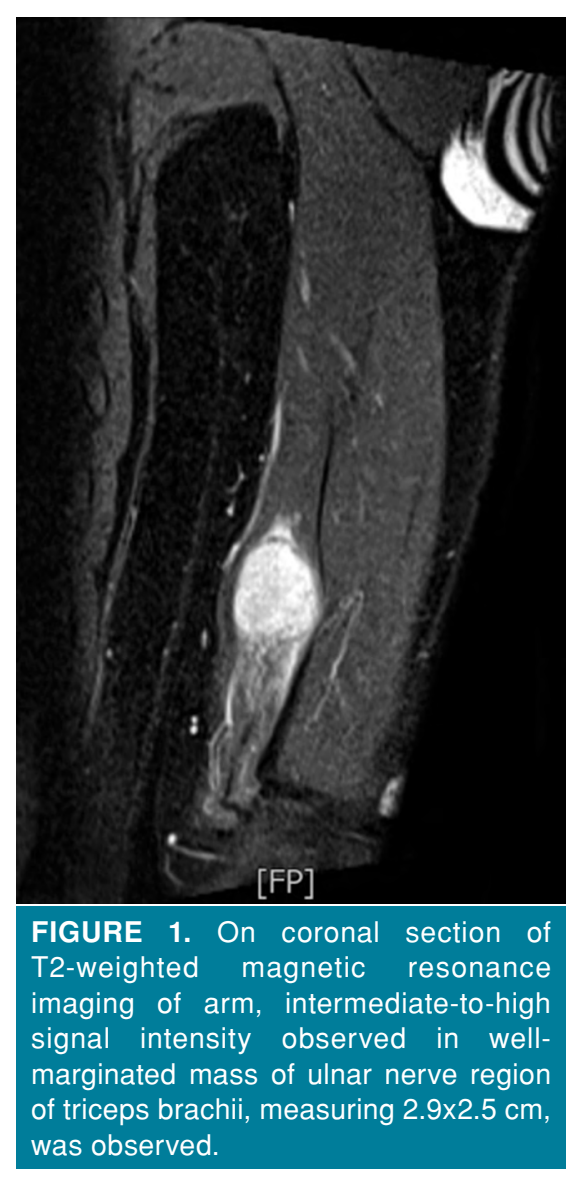

in another institution, a well-marginated mass in the area of the ulnar nerve region of the triceps brachii, measuring $2.9 \times 2.5 \mathrm{~cm}$, was observed. On T2-weighted imaging, intermediate-to-high signal intensity
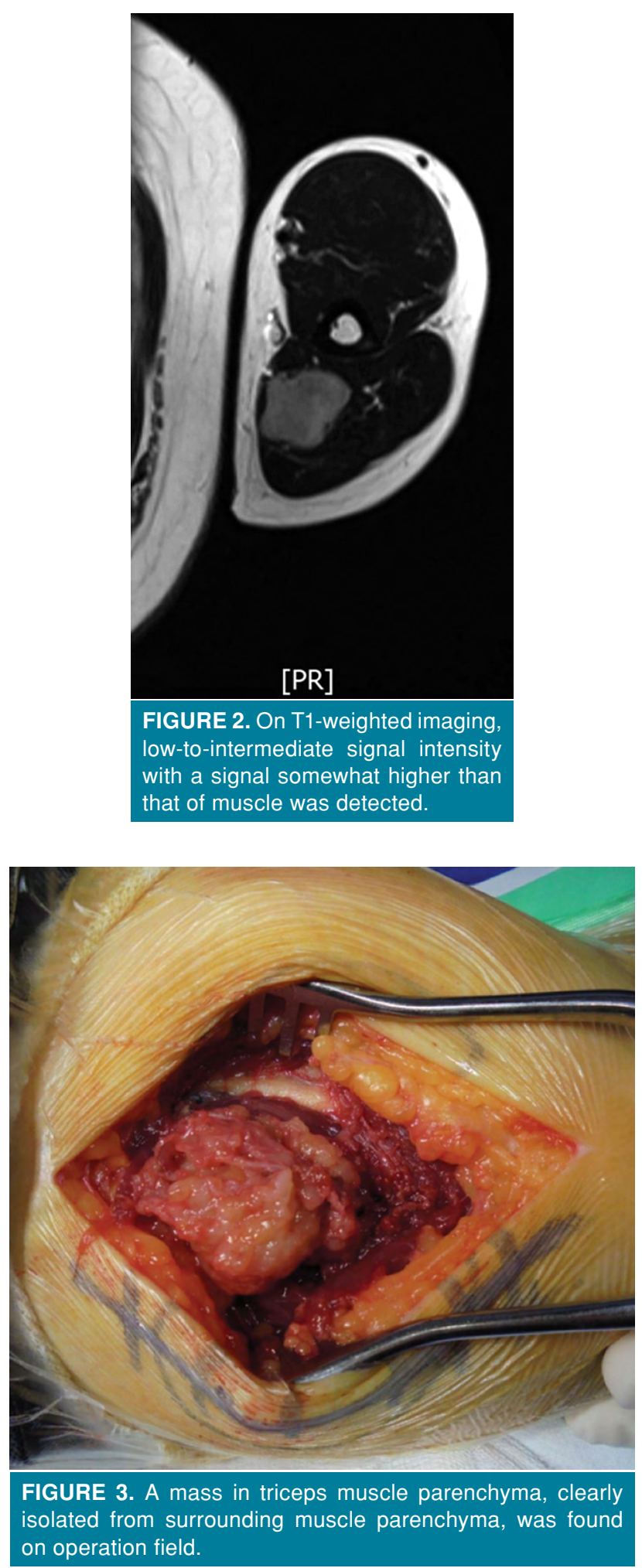

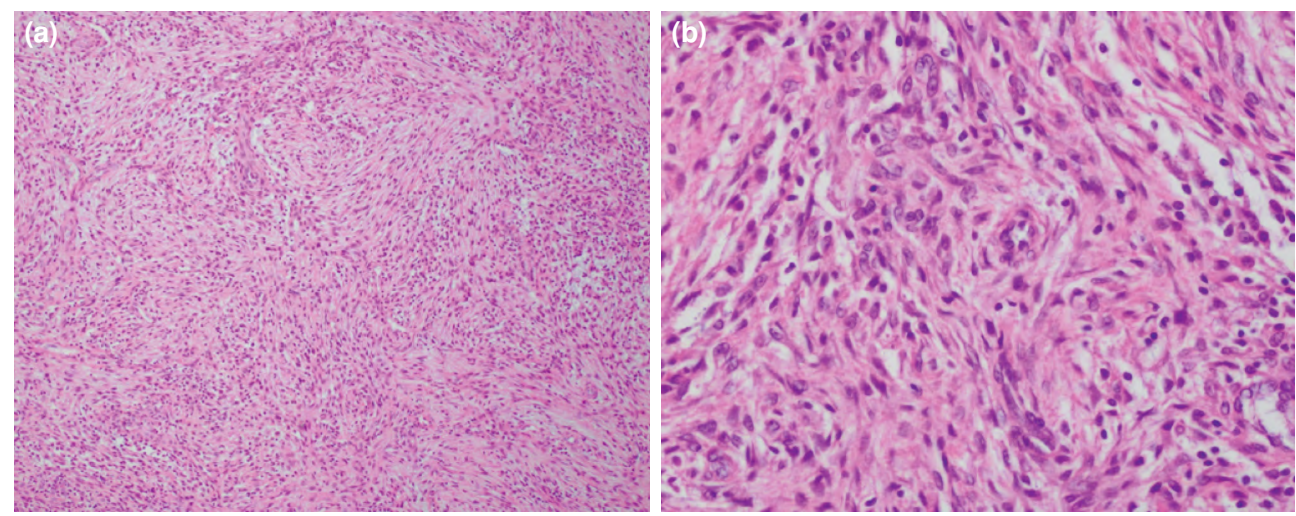

FIGURE 4. (a) Under light microscopy, myofibroblastic spindle cells, myofibroblasts, collagen, various plasma cells, lymphocytes, and histocytes were observed $(H-E \times 40)$. (b) Spindle cells had an abundant cytoplasm and formed loose bundles of fibers in different directions. Small amounts of collagen were deposited in between, and owing to frequent infiltrations, density of cells was not particularly high, and no dysplasia of spindle cells was detected $(H-E \times 400)$.

was observed (Figure 1). On T1-weighted imaging, low-to-intermediate signal intensity with a signal somewhat higher than that of the muscle was detected (Figure 2). An operation was performed, and a mass in the triceps muscle parenchyma, clearly isolated from the surrounding muscle parenchyma, was found.
No infiltration into the parenchyma of the muscle was observed (Figure 3). As the mass compressed the ulnar nerve, a wide excision was performed with attention to the ulnar nerve. Under light microscopy, myofibroblastic spindle cells, myofibroblasts, collagen, various plasma cells, lymphocytes, and histocytes
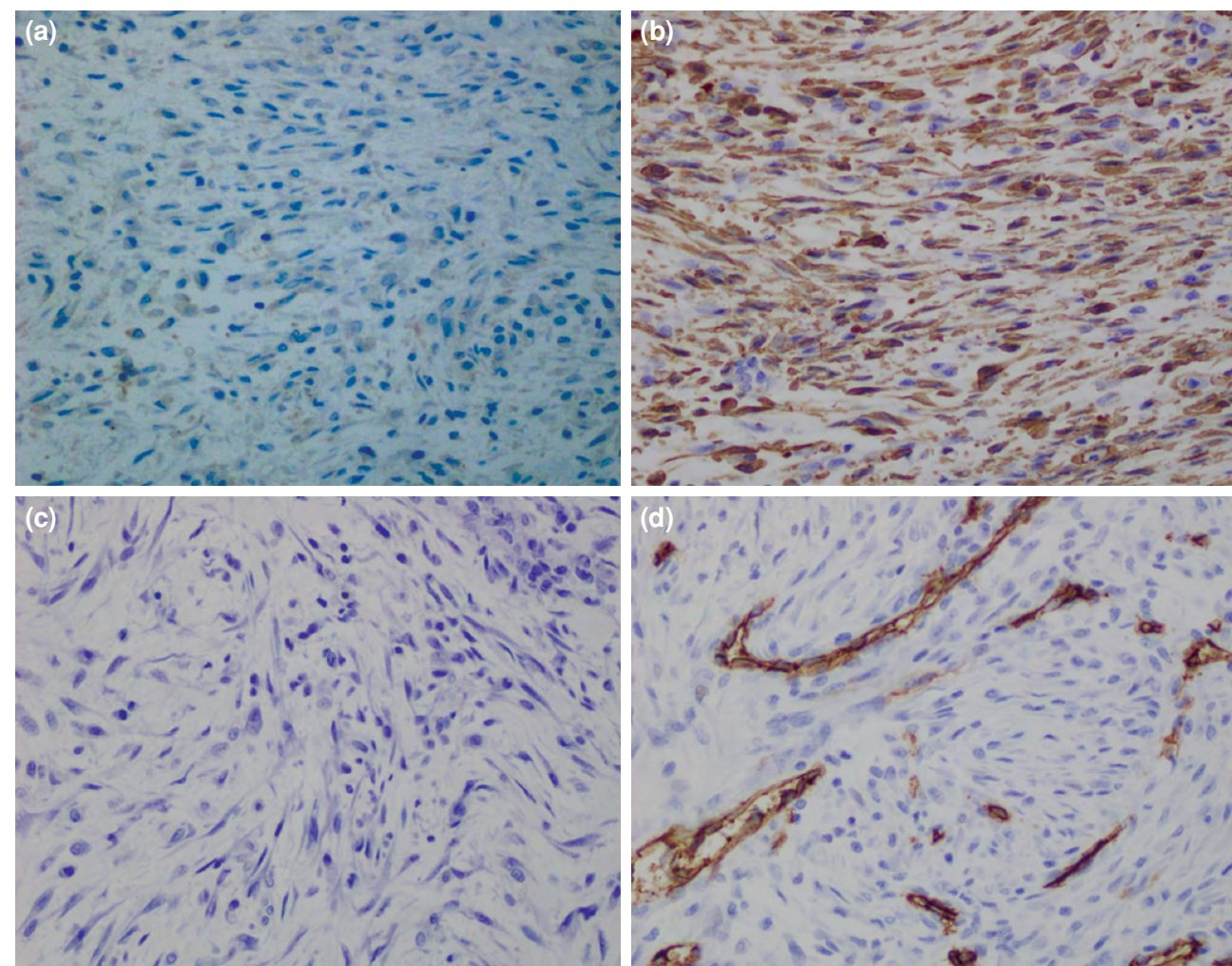

FIGURE 5. Immunohistochemical staining for differentiation showed spindle cells reacting negatively to VENTANA anaplastic lymphoma kinase immunohistochemistry (D5F3) (a) and positively to smooth muscle actin (b), S-100 protein (c), and cluster of differentiation 34 (d). 
were observed (Figure 4a). The spindle cells had an abundant cytoplasm and formed loose bundles of fibers in different directions. Small amounts of collagen were deposited in between, and owing to frequent infiltrations, the density of the cells was not particularly high, and no dysplasia of spindle cells was detected (Figure 4b). The immunohistochemical staining for differentiation showed spindle cells reacting negatively to VENTANA ALK immunohistochemistry (D5F3) (F. Hoffmann-La Roche, Basel, Switzerland) (Figure 5a) and positively to smooth muscle actin (SMA) (Figure 5b), S-100 protein (Figure $5 \mathrm{c}$ ), and cluster of differentiation 34 (Figure 5d). Thus, a diagnosis of ALK-negative IMT was established. Pathological examination revealed that the tumor margin was free of tumor cells. All symptoms of the patient due to ulnar nerve compression were improved. No recurrence was observed in the last 12 months, and the patient's condition is currently being followed-up with observation. A written informed consent was obtained from the patient.

\section{DISCUSSION}

The IMTs are most prevalent in the lungs. As IMTs in the lungs have a mild clinical progression with rare metastases and relapses, they were once called "inflammatory pseudotumors" because they do not have the characteristics of typical tumors. ${ }^{[1,12]}$ In comparison, Inflammatory myofibroblastic tumors in organs outside of the lungs are known to be relatively rare, have poor prognosis, and occur in various organs. The prognosis is known to depend on the area in which the tumor occurs. Coffin et al. ${ }^{[13]}$ reported 84 cases of extrapulmonary IMTs, of which three $(3 \%)$ occurred in the extremities. Maschiocci et al. ${ }^{[14]}$ reported seven cases of IMTs in the extremities. Inflammatory myofibroblastic tumors in the limbs have only been reported in only one or two cases; hence, little is known about the incidence and prognosis of the tumors. ${ }^{[15,16]}$

In this tumor, fibroblasts and myofibroblasts forming a cell bundle proliferate and are accompanied by the infiltration of chronic inflammatory cells. Immunohistochemical staining showed myofibroblasts reacting positively to vimentin and SMA in particular, reacting partially positively to desmin and cytokeratin, and expressing the ALK-1 protein (p80). Recent cytogenetic studies of inflammatory myofibroblastoma have revealed that about half of these tumors are caused by a clone rearrangement at the chromosome $2 \mathrm{p} 23$ site. The chromosome 2p23 site contains the ALK gene, and if this site is mutated, the IMT expresses ALK. A mutation in ALK was first documented in anaplastic large cell lymphoma (ALCL). ALK-positive ALCLs and IMTs have a better prognosis. ${ }^{[17]}$ These findings suggest that IMT is neoplastic. Reports regarding the disease have described cases in which trauma, surgery, autoimmune reactions, infections, EpsteinBarr virus, and other factors were the causes, but in most cases, the exact cause and etiology were unknown. ${ }^{[13,18]}$ The patient in the present case also had no history that could have caused the disease.

Only few reports have described malignant changes of IMTs in the soft tissues of the extremities. On the basis of our literature search, only Donner et al. ${ }^{[19]}$ performed a surgical resection of IMTs in the soft tissues of the forearm and reported one case with a 12-year follow-up in which five relapses occurred, after which malignant changes occurred.

In this case, the borderline was clear, but the characteristics of a histologically low-grade malignant tumor were detected. Furthermore, immunohistochemical staining was positive for SMA but negative for ALK, which led to the diagnosis of ALK-negative IMT. As reported in the literature, inflammatory myoblastoma (inflammatory fibrosarcoma) lacks morphological factors and has a relatively low proliferative index, making it either a tumor with an unpredictable prognosis or a low-grade sarcoma; hence, total tumor resection and long-term regular follow-up are needed. ${ }^{[20,21]}$ In the present case, a wide excision was performed for the IMT, and outpatient follow-up was conducted. No widely accepted theory exists, and research is needed regarding the effects of adjuvant treatment after the surgical resection of IMT. In addition, further research is needed on whether inflammatory fibroblastoma and inflammatory fibrosarcoma are the same or different tumors with some morphological similarities.

Declaration of conflicting interests

The authors declared no conflicts of interest with respect to the authorship and/or publication of this article.

\section{Funding}

The authors received no financial support for the research and/or authorship of this article.

\section{REFERENCES}

1. Umiker WO, Iverson L. Postinflammatory tumors of the lung; report of four cases simulating xanthoma, fibroma, or plasma cell tumor. J Thorac Surg 1954;28:55-63.

2. Scott L, Blair G, Taylor G, Dimmick J, Fraser G. Inflammatory pseudotumors in children. J Pediatr Surg 1988;23:755-8. 
3. Coffin CM, Humphrey PA, Dehner LP. Extrapulmonary inflammatory myofibroblastic tumor: a clinical and pathological survey. Semin Diagn Pathol 1998;15:85-101.

4. Lin J, Liu H, Zhuang Y, Yang P, Zheng Y, Yang Y, et al. Inflammatory myofibroblastic tumor of the thigh without bone involvement: a case report. World J Surg Oncol 2014;12:208.

5. Savvidou OD, Sakellariou VI, Papakonstantinou O, Skarpidi E, Papagelopoulos PJ. Inflammatory myofibroblastic tumor of the thigh: presentation of a rare case and review of the literature. Case Rep Orthop 2015;2015:814241.

6. Li J, Yin WH, Takeuchi K, Guan H, Huang YH, Chan JK. Inflammatory myofibroblastic tumor with RANBP2 and ALK gene rearrangement: a report of two cases and literature review. Diagn Pathol 2013;8:147.

7. Applebaum H, Kieran MW, Cripe TP, Coffin CM, Collins $\mathrm{MH}$, Kaipainen A, et al. The rationale for nonsteroidal anti-inflammatory drug therapy for inflammatory myofibroblastic tumors: a Children's Oncology Group study. J Pediatr Surg 2005;40:999-1003.

8. Mai S, Xiong G, Diao D, Wang W, Zhou Y, Cai R. Case report: Crizotinib is effective in a patient with ROS1-rearranged pulmonary inflammatory myofibroblastic tumor. Lung Cancer 2019;128:101-4.

9. Watanabe H, Uruma T, Tazaki G, Tajiri T, Kikuchi R, Itoh $\mathrm{M}$, et al. Remission of ALK-negative primary pulmonary inflammatory myofibroblastic tumor on treatment with clarithromycin: A case report and review of the literature. Oncol Lett 2016;11:1757-61.

10. Atik OŞ. Is there something new and interesting in my article? Eklem Hastalik Cerrahisi 2019;30:69.

11. Narla LD, Newman B, Spottswood SS, Narla S, Kolli R. Inflammatory pseudotumor. Radiographics 2003;23:719-29.

12. Sakurai H, Hasegawa T, Watanabe Si, Suzuki K, Asamura $\mathrm{H}$, Tsuchiya R. Inflammatory myofibroblastic tumor of the lung. Eur J Cardiothorac Surg 2004;25:155-9.

13. Coffin CM, Watterson J, Priest JR, Dehner LP.
Extrapulmonary inflammatory myofibroblastic tumor (inflammatory pseudotumor). A clinicopathologic and immunohistochemical study of 84 cases. Am J Surg Pathol 1995;19:859-72.

14. Masciocchi C, Lanni G, Conti L, Conchiglia A, Fascetti E, Flamini S, et al. Soft-tissue inflammatory myofibroblastic tumors (IMTs) of the limbs: potential and limits of diagnostic imaging. Skeletal Radiol 2012;41:643-9.

15. Watanabe K, Tajino $T$, Sekiguchi M, Suzuki T. Inflammatory myofibroblastic tumor (inflammatory fibrosarcoma) of the bone. Arch Pathol Lab Med 2000;124:1514-7.

16. Horger M, Müller-Schimpfle M, Wehrmann M, Mehnert B, Maurer F, Eschmann SM, et al. Giant bilateral inflammatory pseudotumor arising along the arterial sheath of the lower extremities. AJR Am J Roentgenol 2002;179:637-9.

17. Mergan F, Jaubert F, Sauvat F, Hartmann O, Lortat-Jacob S, Révillon $Y$, et al. Inflammatory myofibroblastic tumor in children: clinical review with anaplastic lymphoma kinase, Epstein-Barr virus, and human herpesvirus 8 detection analysis. J Pediatr Surg 2005;40:1581-6.

18. Wenig BM, Devaney $\mathrm{K}$, Bisceglia M. Inflammatory myofibroblastic tumor of the larynx. A clinicopathologic study of eight cases simulating a malignant spindle cell neoplasm. Cancer 1995;76:2217-29.

19. Donner LR, Trompler RA, White RR 4th. Progression of inflammatory myofibroblastic tumor (inflammatory pseudotumor) of soft tissue into sarcoma after several recurrences. Hum Pathol 1996;27:1095-8.

20. Meis-Kindblom JM, Kjellström C, Kindblom LG. Inflammatory fibrosarcoma: update, reappraisal, and perspective on its place in the spectrum of inflammatory myofibroblastic tumors. Semin Diagn Pathol 1998;15:133-43.

21. Coffin CM, Dehner LP, Meis-Kindblom JM. Inflammatory myofibroblastic tumor, inflammatory fibrosarcoma, and related lesions: an historical review with differential diagnostic considerations. Semin Diagn Pathol 1998;15:102-10. 